\title{
The Enteric Nerve System as Target of Regulated and Emerging Food-Associated Mycotoxins
}

\author{
Michał Dąbrowski ${ }^{1}$, Hamza Olleik ${ }^{2}$, Amine Kadri ${ }^{2}$, Valérie Camps ${ }^{2}$, Josette Perrier ${ }^{2}$, Philippe Pinton ${ }^{3}$, Isabelle \\ P. Oswald ${ }^{3}$, Lukasz Zielonka ${ }^{1}$, Marc Maresca ${ }^{2, *}$ \\ 1 Department of Veterinary Prevention and Feed Hygiene, Faculty of Veterinary Medicine, University of \\ Warmia and Mazury in Olsztyn, Oczapowski Str. 13/29, 10-718 Olsztyn, Poland; \\ michal.dabrowski@uwm.edu.pl (M.D.); lukaszz@uwm.edu.pl (Ł.Z.) \\ 2 Aix Marseille University., CNRS, Centrale Marseille, iSm2, 13397 Marseille, France; hamza.olleik@live.com \\ (H.O.); keops1986@icloud.com (A.K.); camps.valerie@orange.fr (V.C.); josette.perrier@univ-amu.fr (J.P.) \\ 3 Toxalim, Research center in Food Toxicology, Université de Toulouse, INRAE, ENVT, INP- PURPAN, UPS, \\ F-31027 Toulouse, France; Philippe.Pinton@inrae.fr (P.P.); isabelle.oswald@inrae.fr (I.P.O.) \\ * Correspondence: m.maresca@univ-amu.fr; Tel.: +33-413-945-609
}

Citation: Dąbrowski, M.; Olleik, H. Kadri, A.; Camps, V.; Perrier, J.; Pinton, P.; Oswald, I.P.; Zielonka,Ł.; Maresca, M. The Enteric Nerve System as Target of Regulated and Emerging Food-Associated Mycotoxins. Toxins 2021, 13, $\mathrm{x}$ https://doi.org/10.3390/xxxxx

Received: 29 October 2020 Accepted: 11 January 2021 Published: 14 January 2021

Publisher's Note: MDPI stays neutral with regard to jurisdictional claims in published maps and institutional affiliations.

Copyright: (C) 2021 by the authors. Licensee MDPI, Basel, Switzerland. This article is an open access article distributed under the terms and conditions of the Creative Commons Attribution (CC BY) license (http://creativecommons.org/licenses /by/4.0/).

\begin{abstract}
Food and feed are frequently contaminated by numerous regulated and emerging mycotoxins. Humans and animals are thus exposed daily to mycotoxins through the oral route making of the gut the first and the more exposed tissue. Although many studies have evaluated and demonstrated the impact of mycotoxins on the intestinal epithelial cells (IECs) and on the brain cells, surprisingly only few studies have investigated their impact on cells of the enteric nerve system (ENS). In the present work, we measured the impact of major regulated and emerging mycotoxins (18 mycotoxins in total) on the proliferation and viability of normal rat enteric glial cells (EGCs) in vitro. On the 18 mycotoxins tested, 12 were found toxic with anti-proliferative and/or cytotoxic effects observed at doses ranging from 0.19 to $118 \mu \mathrm{M}$ and 0.4 to $59.59 \mu \mathrm{M}$, respectively. It can be concluded that alterations of the EGCs caused by at least some mycotoxins may participate in their global impact on the gut and the full organism.
\end{abstract}

Keywords: food safety; food contaminants; mycotoxins; emerging mycotoxins; enteric nerve system; enteric glial cells; cyclohexadepsipeptide

Key Contribution: Mycotoxins are able to affect proliferation and viability of enteric glial cells suggesting the implication of alteration of ENS in mycotoxicosis.

\section{Introduction}

Mycotoxins are deleterious secondary metabolites produced by various molds belonging mainly to Aspergillus, Penicillium or Fusarium species and able to colonize plants and crops [1-4]. Food and feed are thus frequently contaminated by numerous regulated and emerging mycotoxins, humans and animals being exposed daily to those toxins through the oral route $[5,6]$. As a consequence, the intestine is the first and the more exposed tissue to food-associated mycotoxins. The impact of mycotoxins (regulated and emerging ones) on the viability and functions of intestinal epithelial cells (IECs) has been extensively investigated (for review [7-14]). Similarly, although less data are available compared to IECs, the impact of mycotoxins on viability and functions of brain cells has been evaluated and described (for review [12,15-21]). Although the enteric nerve system (ENS) similarly to IECs may be exposed to high doses of mycotoxins, surprisingly only few studies have investigated the impact of those toxins on cells of this system ([22-24] and for review [25]). Even less is known about the impact of mycotoxins on enteric glial cells (EGCs). EGCs are key players in the ENS formation, homeostasis and functions as 
well as more generally in gut physiology. For example, the crosstalk between EGCs and IECs plays a role in the establishment and maintain of the intestinal barrier and immune functions (for review [26-31]). In their pioneer study, Rissato et al. using rats as in vivo model were the first and only one to evaluate and demonstrate the impact of a mycotoxin (i.e., deoxynivalenol) on EGCs [32]. Based on that ascertainment, in the present work, we evaluated in vitro the impact on normal rat EGCs of 18 mycotoxins (regulated or emerging ones) known or suspected to be toxic for humans or animals and frequently found in food and feed [5]. The 6 regulated toxins tested were aflatoxin B1 (AFB1), deoxynivalenol (DON), fumonisin B1 (FB1), ochratoxin A (OTA), patulin (PAT), and zearalenone (ZEA). The 12 emerging mycotoxins tested were apicidin (API), aurofusarin (AFN), beauvericin (BEA), brevianamide-F (BRV-F), cyclo-(L-Pro-L-Tyr) (CYCLO), emodin (EMO), enniatins (ENNs) (A, A1, B, B1), moniliformin (MON), and tryptophol (TRPT). Data shown that 12 out of the 18 mycotoxins tested are toxic to normal rat EGCs affecting their division and/or viability at low to high doses, suggesting a potential role for mycotoxin-induced alterations of EGCs in mycotoxicosis, API, DON, and cyclohexadepsisptide mycotoxins (i.e., ENNs and BEA) being the more toxic.

\section{Results}

\subsection{Impact of Regulated and Emerging Mycotoxins on EGC Proliferation}

In a first series of experiments, anti-proliferative effect of mycotoxins was evaluated. Dividing normal rat EGCs were exposed to increasing concentrations of mycotoxins for $48 \mathrm{~h}$ before estimation of cell density using resazurin assay and determination of the $\mathrm{IC}_{50}$ (Figure 1 and Table 1). Among the 6 regulated mycotoxins tested, only 4, i.e., DON, OTA, PAT, and ZEA were able to inhibit the division of normal rat EGCs with IC50 values of $0.19+/-0.07 ; 17.53+/-7.78 ; 10.78+/-1.38$ and $118+/-5.75 \mu \mathrm{M}$, respectively. The two other regulated mycotoxins tested (i.e., AFB1 and FB1) were found inactive, even at $100 \mu \mathrm{M}$ (Figure 1A). The cyclohexadepsipeptides ENNs and BEA were all found very active with IC50 values ranging from $0.92+/-1.07$ to $1.41+/-0.20 \mu \mathrm{M}$ (Figure 1B). Finally, regarding the other emerging mycotoxins, AFN, API and EMO were found anti-proliferative with IC 50 values of $79.51+/-3.68 ; 1.25+/-0.17$ and $40.01+/-2.70 \mu \mathrm{M}$, respectively, whereas CYCLO, BRV-F, MON and TRPT were inactive up to $100 \mu \mathrm{M}$ (Figure 1C).

Table 1. Inhibitory effect of mycotoxins on proliferation and viability of normal rat EGCs. Normal rat EGCs were exposed to increasing concentrations of mycotoxins for $48 \mathrm{~h}$ before determination of their inhibitory effect on proliferation or cell viability. IC 50 values were determined from Figures 1 and 2 using GraphPad ${ }^{\circledR}$ Prism 7 and are expressed as means +/- S.D (in $\mu \mathrm{M}$ ).

\begin{tabular}{|c|c|c|c|c|c|c|}
\hline & AFB1 & DON & FB1 & OTA & PAT & ZEA \\
\hline Proliferation & $>100$ & $0,19+/-0.07$ & $>100$ & $17.53+/-7.78$ & $10,78+/-1.38$ & $118+/-5.75$ \\
\hline \multirow[t]{2}{*}{ Viability } & $>100$ & $5.06+/-0.48$ & $>100$ & $23.88+/-1.36$ & $38.02+/-11.37$ & $31.75+/-4.94$ \\
\hline & BEA & ENN A & ENN A1 & ENN B & ENN B1 & CYCLO \\
\hline Proliferation & $1.41+/-0.20$ & $0.93+/-0.13$ & $1.08+/-0.34$ & $1.40+/-0.18$ & $0.92+/-1.07$ & $>100$ \\
\hline \multirow[t]{2}{*}{ Viability } & $1.91+/-0.45$ & $1.05+/-0.11$ & $0.86+/-0.09$ & $0.72+/-0.16$ & $2.14+/-0.17$ & $>100$ \\
\hline & AFN & API & BRV-F & EMO & MON & TRPT \\
\hline Proliferation & $79.51+/-3.68$ & $1.25+/-0.17$ & $>100$ & $40.01+/-2.70$ & $>100$ & $>100$ \\
\hline Viability & $>100$ & $59.59+/-10.27$ & $>100$ & $>100$ & $>100$ & $>100$ \\
\hline
\end{tabular}


A

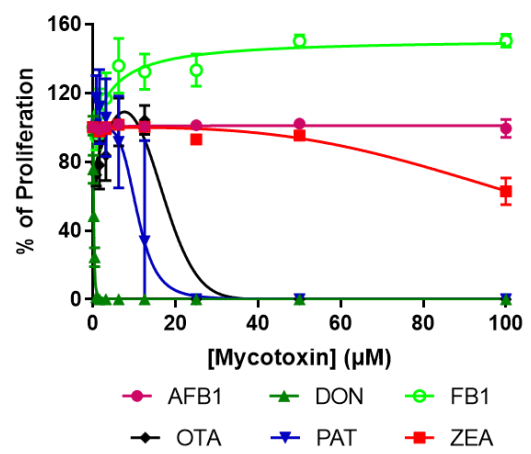

B

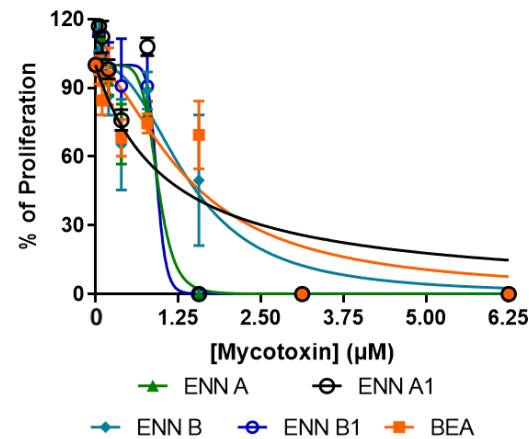

C

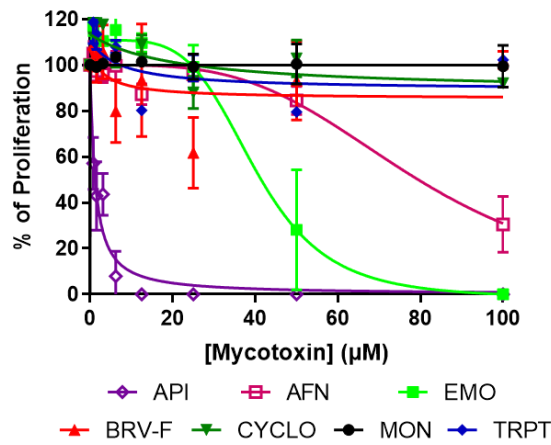

Figure 1. Anti-proliferative effect of mycotoxins on normal rat EGCs. Dividing normal rat EGCs were exposed to increasing concentrations of regulated (A) or emerging mycotoxins (B, C) for $48 \mathrm{~h}$ before evaluation of the cell number using

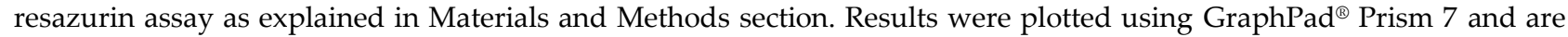
expressed as percentage of proliferation, the negative controls (vehicle alone) giving $100 \%$ proliferation $(n=3)$. .

\subsection{Impact of major regulated and emerging mycotoxins on EGC viability}

The cytotoxic effect of mycotoxins was then measured using non-dividing cells. Confluent normal rat EGCs were exposed for $48 \mathrm{~h}$ to increasing concentrations of mycotoxins before estimation of the number of living cells using resazurin assay and determination of the IC $\mathrm{C}_{50}$ values (Figure 2 and Table 1). Regarding regulated mycotoxins (Figure 2A), only DON, OTA, PAT, and ZEA were found cytotoxic to EGCs with IC 50 of $5.06+/-0.48$; $23.88+/-1.36 ; 38.02+/-11.37$ and $31.75+/-4.94 \mu \mathrm{M}$, respectively. Among the 12 emerging mycotoxins tested (Figure 2B, 2C), only API (IC50 value of $59.59+/-10.27 \mu \mathrm{M}$ ) and the cyclohexadepsipeptides (ENNs and BEA) (with IC 50 values ranging from $0.72+/-0.16$ to $2.14+/-0.17 \mu \mathrm{M})$ were found cytotoxic. The other regulated or emerging mycotoxins tested (i.e., AFB1, AFN, BRV-F, CYCLO, EMO, FB1, MON, and TRPT) were all found not toxic up to $100 \mu \mathrm{M}$.

A

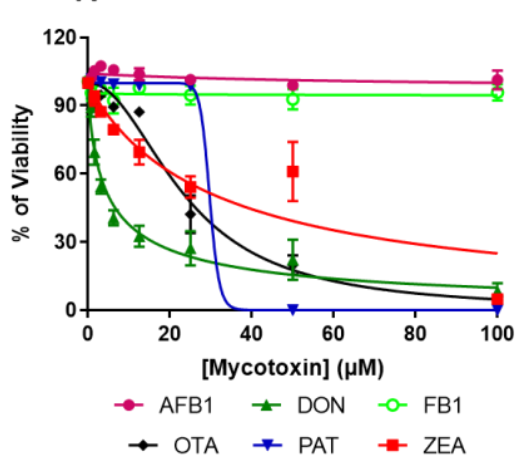

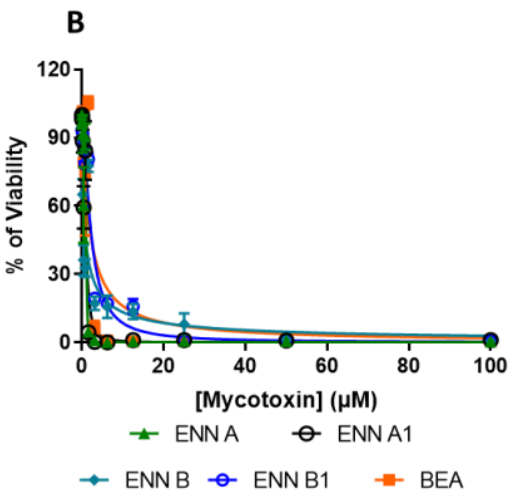

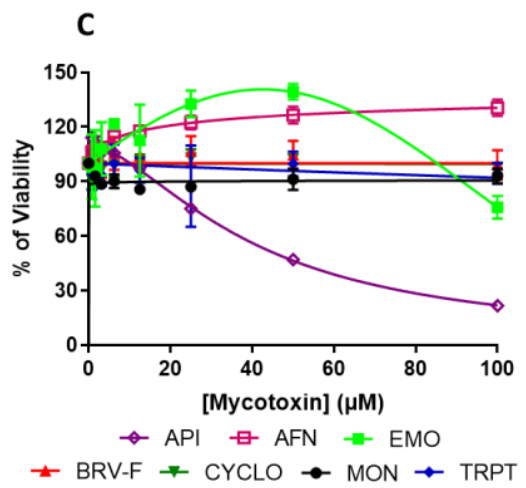

Figure 2. Cytotoxic effect of mycotoxins on normal rat EGCs. Confluent normal rat EGCs were exposed to increasing concentrations of regulated (A) or emerging mycotoxins (B, C) for $48 \mathrm{~h}$ before evaluation of the living cell number using

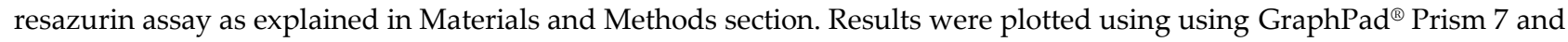
are expressed as percentage of viability, the negative controls (vehicle alone) giving $100 \%$ viability $(n=3)$.

\section{Discussion}

In the present study, we evaluated the toxicity of 18 regulated or emerging mycotoxins on enteric glial cells The eighteen toxins used were selected on the basis of their occurrence in food and feed and of their known or suspected toxicity for humans and/or animals [5]. The regulated mycotoxins tested were AFB1, DON, FB1, OTA, PAT and ZEA whereas emerging mycotoxins tested were API, AFN, BEA, BRV-F, CYCLO, EMO, ENNs (ENN A, A1, B, B1), MON, and TRPT. Among the 18 mycotoxins tested, 12 toxins were 
able to inhibit or suppress the proliferation of normal rat EGCs, i.e., DON, OTA, PAT, and ZEA for the regulated ones and API, AFN, BEA, EMO, ENNs (ENN A, A1, B and B1) for the emerging ones. Anti-proliferative effect was observed at doses ranging from 0.19 to $118 \mu \mathrm{M}$ with the following order of IC 50 : DON $<$ ENN A, A1, B1 $<$ API $<$ ENN B $=$ BEA $<$ PAT $<$ OTA $<$ EMO $<$ AFN $<$ ZEA (Table 1 ).

Regarding cytotoxicity, 10 mycotoxins were able to decrease the viability of normal rat EGCs, i.e., DON, OTA, PAT, and ZEA for the regulated ones and API, BEA, ENNs (ENN A, A1, B and B1) for the emerging ones. Cytotoxic effect was observed at doses ranging from 0.4 to $59.59 \mu \mathrm{M}$ with the following order of IC50: ENN B $<$ ENN A1 $<$ ENN A $<$ BEA $<$ ENN B1 < DON < OTA < PAT < API (Table 1). AFB1, BRV-F, CYCLO, FB1, TRPT, and MON were all found not toxic to normal rat EGCs at least for doses up to 100 $\mu \mathrm{M}$ and after $48 \mathrm{~h}$ treatment. Sensitivity of normal rat EGCs to mycotoxins depends on their status, i.e., proliferating / dividing or confluent / non-dividing cells. OTA, BEA and ENNs (ENN A, A1, B1) gave very similar IC 50 values on dividing and non-dividing cells with 0.77 to 1.36 -fold difference in IC 50 between dividing and non-dividing EGCs. Oppositely, API was more active on dividing than non-dividing cells with $\mathrm{IC}_{50}$ of 1.25 and 59.59 $\mu \mathrm{M}$, respectively (47.6-fold difference). AFN, EMO, DON, and PAT gave the same tendency, those toxins being more active on dividing cells than on non-dividing ones (with a 3.5 and 26.6-fold difference for PAT and DON, respectively). Surprisingly, ENN B and ZEA were found more toxic for non-dividing cells than for dividing ones (1.94 and 3.71fold difference, respectively). The reason of such differences could be related to difference in mycotoxin entry and/or detoxification or modulation of the expression of their molecular target(s) in dividing versus non-dividing EGCs. Future works will be necessary to investigate this point and as well as to identify by which mechanism(s) mycotoxins cause toxic effect on EGCs. Mycotoxins such as DON and OTA are known to alter the functions of brain glial cells (i.e., astrocytes) at sub-toxic doses [18,19]. Based on the fact that astrocytes and EGCs share many common features in term of sensitivity to drugs and physiological functions [30], it will be worthwhile to evaluate if sublethal doses of mycotoxins affect EGCs functions as observed with phycotoxins [33].

\section{Conclusions}

In conclusion, our data confirmed the hypothesis of Gonkowski et al. [25] about a potential alteration of ENS by at least some food-associated mycotoxins. Our data generated using rat EGCs in vitro confirmed the pioneer work of Rissato et al. [32] that showed that deoxynivalenol is able to affect proliferation and viability of EGCs in vivo in rats. In addition, our in vitro data demonstrated that 11 other regulated or emerging mycotoxins (i.e., API, AFN, EMO, ENNs (ENN A, A1, B, B1), BEA, OTA, PAT, and ZEA), are toxic for normal rat EGCs. API, DON, and cyclohexadepsipetides mycotoxins (i.e., ENNs and BEA) are the more worrying toxins with $\mathrm{IC}_{50}$ as low as 0.4 to $5 \mu \mathrm{M}$, similar tendency being observed with IECs [5]. This study demonstrated that EGCs are the target of at least some food-associated mycotoxins. Due to their pivotal role in the gut physiology [26-31], the toxic effect of mycotoxins on EGCs could lead to alterations of both ENS and IECs homeostasis and functions, further reinforcing the hypothesis, first enounced in 2010, of a link between mycotoxins and inflammatory bowel diseases (IBD) [35]. Future works, including the evaluation of the effects of sub-toxic doses of mycotoxins on key functions of EGCs, will help confirming this hypothesis.

\section{Materials and Methods}

\subsection{Mycotoxins}

Mycotoxins were used in this study were selected based on their occurrence in food and of their known or suspected toxicity for humans and/or animals [5]. Aflatoxin B1 (AFB1) (purity $>98 \%$ ), apicidin (API) (purity $>98 \%$ ), beauvericin (BEA) (purity $>97 \%$ ), deoxynivalenol (DON) (purity $>98 \%$ ), emodin (EMO) (purity $>90 \%$ ), enniatins (ENNs) 
(A, A1, B, B1) (purity $>99 \%$ ), fumonisin B1 (FB1) (purity $>98 \%$ ), moniliformin (MON) (purity $>95 \%$ ), ochratoxin A (OTA) (purity $>95 \%$ ), patulin (PAT) (purity $>98 \%$ ), tryptophol (TRPT) (purity > 97\%) and zearalenone (ZEA) (purity > 98\%) were obtained from Sigma Aldrich (Lyon, France). Cyclo-(L-Pro-L-Tyr) (CYCLO) (purity > 98\%), and brevianamide-F (BRV-F) (purity > 95\%) were purchased from BioAustralis (Smithfield, Australia). Aurofusarin (AFN), (purity > 97\%) was purchased from Santa Cruz Biotechnology (Dallas, TX, USA). All mycotoxins were dissolved in ethanol or DMSO following manufacturer's instructions to prepare stock solutions stored at $-20^{\circ} \mathrm{C}$. Working dilutions were freshly prepared in cell culture medium for each experiment.

\subsection{Cell Culture}

Normal rat EGCs (ATCC ${ }^{\circledR}$ CRL2690 ${ }^{\mathrm{TM}}$ ) were obtained from ATCC (LGC Standards Molsheim, France). Cells were cultured in Dulbecco's modified essential medium (DMEM) supplemented with $10 \%$ fetal bovine serum (FBS), $1 \%$ L-glutamine and 1\% antibiotics (from Thermofisher, Illkirch-Graffenstaden, France). Cells were routinely grown on 75 $\mathrm{cm}^{2}$ flasks, maintained in a $5 \% \mathrm{CO}_{2}$ incubator at $37^{\circ} \mathrm{C}$ and passaged when reaching 90 95\% confluence.

\subsection{Anti-Proliferative and Cytotoxicity Assays}

Anti-proliferative and cytotoxic effects were measured as previously described [3540]. Briefly, rat EGCs cells grown on $75 \mathrm{~cm}^{2}$ flasks were detached from flasks using a trypsin-EDTA solution (from Thermofisher, Illkirch-Graffenstaden, France). After counting using a Malassez chamber, cells were diluted in culture media and seeded into 96-well cell culture plates (Greiner bio-one, Paris, France) at the appropriate cell density depending of the assay. For the anti-proliferative assay, rat EGCs were seeded at an initial cell density of approximatively 5000 cells/well in order to let them divide. For the cytotoxicity assay, rat EGCs were seeded at an initial cell density of approximatively 50,000 cells/well in order to rapidly reach confluence. In both cases, after $12 \mathrm{~h}$ at $37^{\circ} \mathrm{C}$ in a $5 \% \mathrm{CO}_{2}$ incubator, wells were empty and the cells were exposed to increasing concentrations of mycotoxins ( 0 to $100 \mu \mathrm{M}, 1 / 2$ dilution) or the corresponding vehicle (i.e., ethanol (maximal volume of $10 \%$ ) or DMSO (maximal volume 1\%), used as negative control and found not toxic) diluted in complete culture media. After $48 \mathrm{~h}$ incubation at $37^{\circ} \mathrm{C}$ in a $5 \% \mathrm{CO}_{2}$ incubator, cell viability was evaluated using resazurin based in vitro toxicity assay kit (Sigma-Aldrich, Lyon, France) following manufacturer's instructions. Briefly, cell wells were empty and cells were treated with $100 \mu \mathrm{L}$ of resazurin diluted 1:10 in sterile phosphate buffer saline (PBS) containing calcium and magnesium $\left(\mathrm{PBS}^{++}, \mathrm{pH} 7.4\right)$. After $4 \mathrm{~h}$ incubation at $37^{\circ} \mathrm{C}$, fluorescence intensity (excitation wavelength of $530 \mathrm{~nm} /$ emission wavelength of $590 \mathrm{~nm}$ ) was measured using microplate reader (Biotek, Synergy Mx, Colmar, France). The fluorescence values were normalized by the controls (vehicle treated cells) and expressed as percent of proliferation or viability. The IC 50 values of mycotoxins on cell proliferation or viability (i.e., the concentration of derivative causing a reduction of $50 \%$ of the cell division or survival) were calculated using GraphPad ${ }^{\circledR}$ Prism 7 software (San Diego, CA, USA). tTest and two ways ANOVA analyses were used to address the significant differences between mean values with significance set at $p<0.05$.

Author Contributions: Conceptualization, M.M.; methodology, M.M.; validation, H.O., M.D., Ł.Z. and M.M.; formal analysis, M.D., H.O., A.K., V.C. and M.M.; investigation, M.M.; data curation, H.O. and M.M.; writing - original draft preparation, M.M.; writing - review and editing, M.D, H.O, A.K., V.C., J.P., P.P., I.P.O., L.K., M.M.; supervision, M.M.; project administration, M.M.; funding acquisition, P.P., I.P.O. and M.M. All authors have read and agreed to the published version of the manuscript.

Funding: This research was funded by Agence Nationale de la Recherche (ANR) through the grant "EmergingMyco", grant number ANR-18-CE34-0014. 
Conflicts of Interest: The authors declare no conflict of interest.

\section{References}

1. Bennett, J.W.; Klich, M. Mycotoxins. Clin. Microbiol. Rev. 2003, 16, 497-516.

2. Frisvad, J.C.; Thrane, U.; Samson, R.A.; Pitt, J.I. Important mycotoxins and the fungi which produce them. Adv. Exp. Med. Biol. 2006, 571, 3-31.

3. Stoev, S.D. Foodborne mycotoxicoses, risk assessment and underestimated hazard of masked mycotoxins and joint mycotoxin effects or interaction. Environ. Toxicol. Pharmacol. 2015, 39, 794-809.

4. Wu, F.; Groopman, J.D.; Pestka, J.J. Public health impacts of foodborne mycotoxins. Annu. Rev. Food Sci. Technol. 2014, 5, 351372.

5. Khoshal, A.K.; Novak, B.; Martin, P.G.P.; Jenkins, T.; Neves, M.; Schatzmayr, G.; Oswald, I.P.; Pinton, P. Co-Occurrence of DON and Emerging Mycotoxins in Worldwide Finished Pig Feed and Their Combined Toxicity in Intestinal Cells. Toxins 2019, 11, 727.

6. Final Report SCOOP Task 3.2.10: Collection of Occurrence Data of Fusarium Toxins in Food and Assessment of Dietary Intake by the Population of EU Member States. Available online: http://ec.europa.eu/food/fs/scoop/task3210.pdf (accessed on 15 February 2016).

7. Alassane-Kpembi, I.; Pinton, P.; Oswald, I.P. Effects of mycotoxins on the intestine. Toxins 2019, 11, 159.

8. Bertero, A.; Fossati, P.; Tedesco, D.E.A.; Caloni, F. Beauvericin and Enniatins: In Vitro Intestinal Effects. Toxins 2020, $12,686$.

9. Gao, Y.; Meng, L.; Liu, H.; Wang, J.; Zheng, N. The Compromised Intestinal Barrier Induced by Mycotoxins. Toxins 2020, 12, 619.

10. Ghareeb, K.; Awad, W.A.; Böhm, J.; Zebeli, Q. Impacts of the feed contaminant deoxynivalenol on the intestine of monogastric animals: Poultry and swine. J. Appl. Toxicol. 2015, 35, 327-337.

11. Maresca, M.; Fantini, J. Some food-associated mycotoxins as potential risk factors in humans predisposed to chronic intestinal inflammatory diseases. Toxicon 2010, 56, 282-294.

12. Maresca, M. From the gut to the brain: Journey and pathophysiological effects of the food-associated trichothecene mycotoxin deoxynivalenol. Toxins 2013, 5, 784-820.

13. Pinton, P.; Oswald, I.P. Effect of deoxynivalenol and other Type B trichothecenes on the intestine: A review. Toxins 2014, 6, 1615-1643.

14. Ren, Z.; Guo, C.; Yu, S.; Zhu, L.; Wang, Y.; Hu, H.; Deng, J. Progress in Mycotoxins Affecting Intestinal Mucosal Barrier Function. Int J Mol Sci. 2019, 20, 2777.

15. Bonnet, M.S.; Roux, J.; Mounien, L.; Dallaporta, M.; Troadec, J.D. Advances in deoxynivalenol toxicity mechanisms: The brain as a target. Toxins 2012, 4, 1120-1138.

16. Doi, K.; Uetsuka, K. Mechanisms of mycotoxin-induced neurotoxicity through oxidative stress-associated pathways. Int J Mol Sci. 2011, 12, 5213-5237.

17. Purzycki, C.B.; Shain, D.H. Fungal toxins and multiple sclerosis: A compelling connection. Brain Res Bull. 2010, 82, 4-6.

18. Razafimanjato, H.; Garmy, N.; Guo, X.J.; Varini, K.; di Scala, C.; di Pasquale, E.; Taïeb, N.; Maresca, M. The food-associated fungal neurotoxin ochratoxin A inhibits the absorption of glutamate by astrocytes through a decrease in cell surface expression of the excitatory amino-acid transporters GLAST and GLT-1. Neurotoxicology 2010, 31, 475-484.

19. Razafimanjato, H.; Benzaria, A.; Taïeb, N.; Guo, X.J.; Vidal, N.; Di Scala, C.; Varini, K.; Maresca, M. The ribotoxin deoxynivalenol affects the viability and functions of glial cells. Glia 2011, 59, 1672-1683.

20. Terciolo, C.; Maresca, M.; Pinton, P.; Oswald, I.P. Review article: Role of satiety hormones in anorexia induction by Trichothecene mycotoxins. Food Chem Toxicol. 2018, 121, 701-714.

21. Zhang, J.; You, L.; Wu, W.; Wang, X.; Chrienova, Z.; Nepovimova, E.; Wu, Q.; Kuca, K. The neurotoxicity of trichothecenes T-2 toxin and deoxynivalenol (DON): Current status and future perspectives. Food Chem Toxicol. 2020, 145, 111676.

22. Makowska, K.; Obremski, K.; Gonkowski, S. The impact of T-2 toxin on vasoactive intestinal polypeptide-like immunoreactive (VIP-LI) nerve structures in the wall of the porcine stomach and duodenum. Toxins 2018, 10, 138.

23. Makowska, K.; Obremski, K.; Zielonka, L.; Gonkowski, S. The influence of low doses of zearalenone and T-2 toxin on calcitonin gene related peptide-like immunoreactive (CGRP-LI) neurons in the ENS of the porcine descending colon. Toxins $2017,9,98$.

24. Obremski, K.; Gonkowski, S.; Wojtacha, P. Zearalenone-induced changes in the lymphoid tissue and mucosal nerve fibers in the porcine ileum. Pol. J. Vet. Sci. 2015, 18, 357-365.

25. Gonkowski, S.; Gajęcka, M.; Makowska, K. Mycotoxins and the Enteric Nervous System. Toxins 2020, $12,461$.

26. Benvenuti, L.; D’Antongiovanni, V.; Pellegrini, C.; Antonioli, L.; Bernardini, N.; Blandizzi, C.; Fornai, M. Enteric Glia at the Crossroads between Intestinal Immune System and Epithelial Barrier: Implications for Parkinson Disease. Int J Mol Sci. 2020, 21, 9199.

27. Neunlist, M.; Rolli-Derkinderen, M.; Latorre, R.; Van Landeghem, L.; Coron, E.; Derkinderen, P.; De Giorgio R. Enteric glial cells: Recent developments and future directions. Gastroenterology 2014, 147, 1230-1237.

28. Ochoa-Cortes, F.; Turco, F.; Linan-Rico, A.; Soghomonyan, S.; Whitaker, E.; Wehner, S.; Cuomo, R.; Christofi, F.L. Enteric Glial Cells: A New Frontier in Neurogastroenterology and Clinical Target for Inflammatory Bowel Diseases. Inflamm Bowel Dis. 2016, $22,433-449$. 
29. Pochard, C.; Coquenlorge, S.; Freyssinet, M.; Naveilhan, P.; Bourreille A.; Neunlist, M.; Rolli-Derkinderen, M. The multiple faces of inflammatory enteric glial cells: Is Crohn's disease a gliopathy? Am J Physiol Gastrointest Liver Physiol. 2018, 315, G1G11.

30. Savidge, T.C.; Sofroniew, M.V.; Neunlist, M. Starring roles for astroglia in barrier pathologies of gut and brain. Lab Invest. 2007, $87,731-736$.

31. Schneider, S.; Wright, C.M.; Heuckeroth, R.O. Unexpected Roles for the Second Brain: Enteric Nervous System as Master Regulator of Bowel Function. Annu Rev Physiol. 2019, 81, 235-259.

32. Rissato, D.F.; de Santi Rampazzo, A.P.; Borges, S.C.; Sousa, F.C.; Busso, C.; Buttow, N.C.; Natali, M.R.M. Chronic ingestion of deoxynivalenol-contaminated diet dose-dependently decreases the area of myenteric neurons and gliocytes of rats. Neurogastroenterol. Motil. 2020;32, e13770.

33. Reale, O.; Huguet, A.; Fessard, V. Novel Insights on the Toxicity of Phycotoxins on the Gut through the Targeting of Enteric Glial Cells. Mar Drugs 2019, 17, 429.

34. Ali, H.; Jabeen, A.; Maharjan, R.; Nadeem-ul-Haque, M.; Aamra, H.; Nazir, S.; Khan, S.; Olleik, H.; Maresca, M.; Shaheen, F. Furan-Conjugated Tripeptides as Potent Antitumor Drugs. Biomolecules 2020, 10, 1684.

35. Maresca, M.; Fantini, J. Some food-associated mycotoxins as potential risk factors in humans predisposed to chronic intestinal inflammatory diseases. Toxicon 2010, 56, 282-294.

36. Borie, C.; Mondal, S.; Arif, T.; Briand, M.; Lingua, H.; Dumur, F.; Gigmes, D.; Stocker, P.; Barbarat, B.; Robert, V.; Nicoletti, C.; Olive, D.; Maresca, M.; Nechab, M. Enediynes bearing polyfluoroaryl sulfoxide as new antiproliferative agents with dual targeting of microtubules and DNA. Eur J Med Chem. 2018, 148, 306-313.

37. Olleik, H.; Yahiaoui, S.; Roulier, B.; Courvoisier-Dezord, E.; Perrier, J.; Pérès, B.; Hijazi, A.; Baydoun, E.; Raymond, J.; Boumendjel, A.; Maresca, M.; Haudecoeur, R. Aurone derivatives as promising antibacterial agents against resistant Gram-positive pathogens. Eur J Med Chem. 2019, 165, 133-141.

38. Olleik, H.; Nicoletti, C.; Lafond, M.; Courvoisier-Dezord, E.; Xue, P.; Hijazi, A.; Baydoun, E.; Perrier, J.; Maresca, M. Comparative Structure-Activity Analysis of the Antimicrobial Activity, Cytotoxicity, and Mechanism of Action of the Fungal Cyclohexadepsipeptides Enniatins and Beauvericin. Toxins 2019, 11, 514.

39. Olleik, H.; Baydoun, E.; Perrier, J.; Hijazi, A.; Raymond, J.; Manzoni, M.; Dupuis, L.; Pauleau, G.; Goudard, Y.; Villéon, B.; Goin, G.; Sockeel, P.; Choudhary, M.I.; Pasquale, E.D.; Nadeem-Ul-Haque, M.; Ali, H.; Khan, A.I.; Shaheen, F.; Maresca, M. TemporinSHa and Its Analogs as Potential Candidates for the Treatment of Helicobacter pylori. Biomolecules 2019, 9, 598.

40. Olleik, H.; Yacoub, T.; Hoffer, L.; Gnansounou, S.M.; Benhaiem-Henry, K.; Nicoletti, C.; Mekhalfi, M.; Pique, V.; Perrier, J.; Hijazi, A.; Baydoun, E.; Raymond, J.; Piccerelle, P.; Maresca, M.; Robin, M. Synthesis and Evaluation of the Antibacterial Activities of 13-Substituted Berberine Derivatives. Antibiotics 2020, 9, 381. 